\title{
Strategies for Improving Safety at Horizontal Curves
}

\author{
Runfen Liu \\ School of Transportation, Shijiazhuang Tiedao Univercity, Shijiazhuang 050043, Hebei, China
}

Keywords: horizontal curves; safety; Collisions

Abstract. Accidents on horizontal curves cause a significant amount of pain and suffering to those involved in the accidents because of the nature of the collisions. Curves are necessary and important elements of every highway. Implementing strategies designed to improve the safety of horizontal curves will help achieve goal of highway safety. The goal of the strategic highway safety is to reduce annual highway fatalities. This goal can be achieved through the widespread application of low-cost, proven countermeasures that reduce the number of crashes on the highways. This paper provides an overall framework for coordinating a safety program. It will be of particular interest to safety practitioners with responsibility for reduce injuries and fatalities on the highway system.

\section{Introduction}

General Description of the Problem. Statistics from the Fatality Analysis Reporting System (FARS) indicate that 42,815 people were killed in 38,309 fatal crashes highway system in 2002

(U.S.) . Approximately 25 percent of these fatalities occurred along horizontal curves (see fig.1). Curves are necessary and important elements of every highway. Considering these statistics and that the average accident rate for horizontal curves is about three times the average accident rate for highway tangents, implementing strategies designed to improve the safety of horizontal curves will help achieve goal of highway safety.

Specific Attributes of the Problem. Accidents on horizontal curves cause a significant amount of pain and suffering to those involved in the accidents because of the nature of the collisions. For example, while only slightly less than 2 percent of all crashes on curved roadway segments are fatal crashes, approximately 40 percent involve some type of injury (see fig.2). Many of the more severe curve-related crashes (i.e., fatal) occur primarily in rural settings. Fig.3 shows that about three-quarters of the fatal crashes occur in rural areas. Fig. 4 shows that more than 70 percent of the fatal crashes occur on secondary roads, fig.5 indicates that attention to the problem of crashes on curves should be directed primarily to two-lane highways in rural areas.

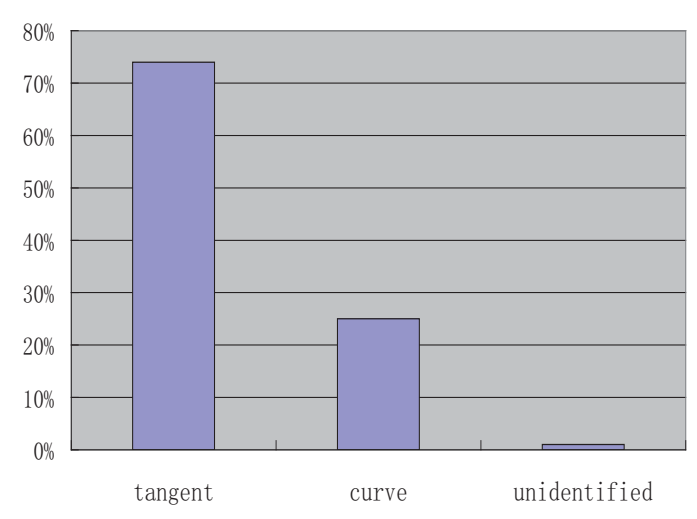

Fig.1: Percentage of fatalities by roadway alignment 


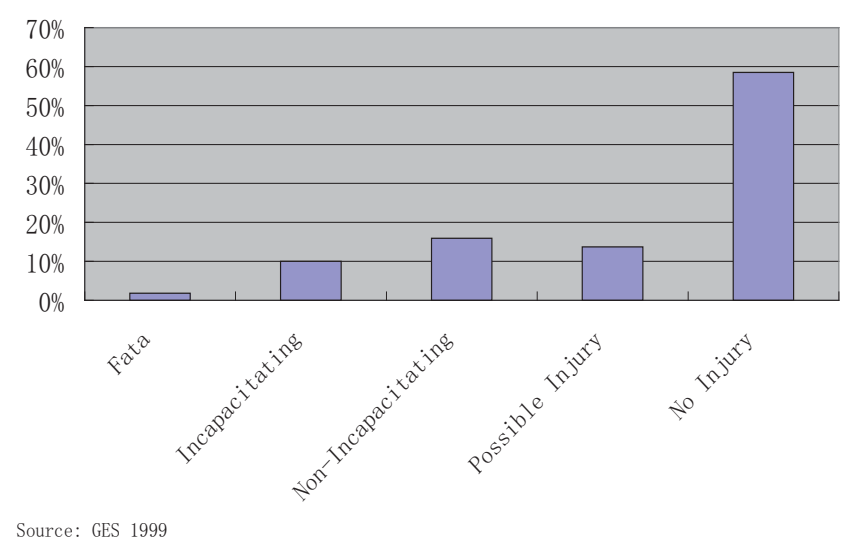

Fig.2: Severity distribution all crashes on curved-roadway segments

The safety of curves is a reflection of both the roadway itself and the roadside environment. Fig.6 which includes crashes of all severities along curved roadway segments (GES, 1999), shows that the first harmful event of a crash on a curved highway segment is just as likely to occur on the traveled way as off the traveled way. (The "Other" category includes locations such as within interchanges.)

The most prevalent types of crashes that occur at horizontal curves are run-off-road (ROR) and head-on crashes. fig. 7 shows that 76 percent of curve-related fatal crashes were single-vehicle crashes in which the vehicle left the roadway and struck a fixed object or overturned, while 11 percent of curve-related fatal crashes were head-on crashes.

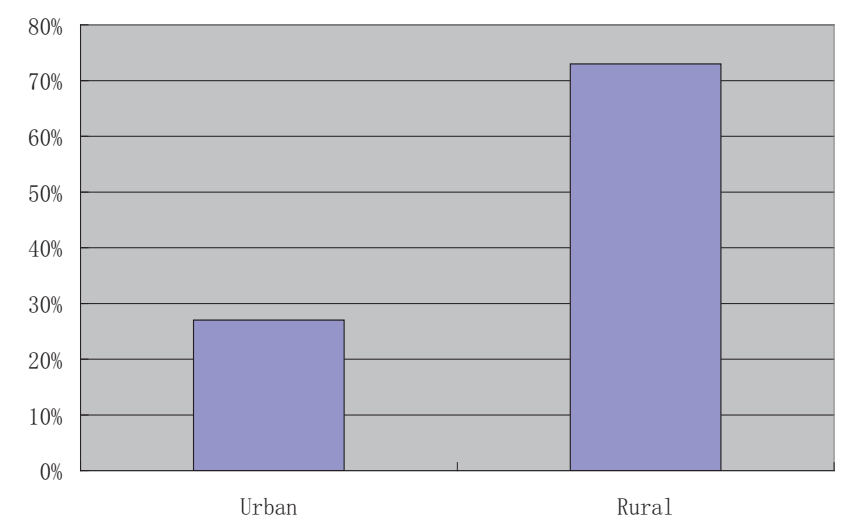

Fig.3: Location of fatal crashes on horizontal curves

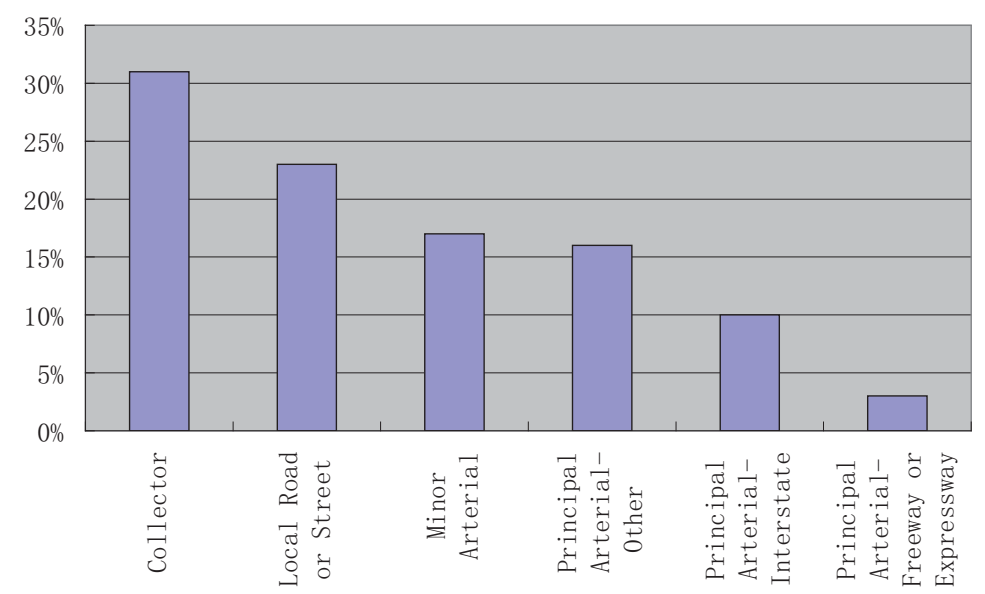

Fig.4: Location of fatal crashes on horizontal curves by roadway classification 


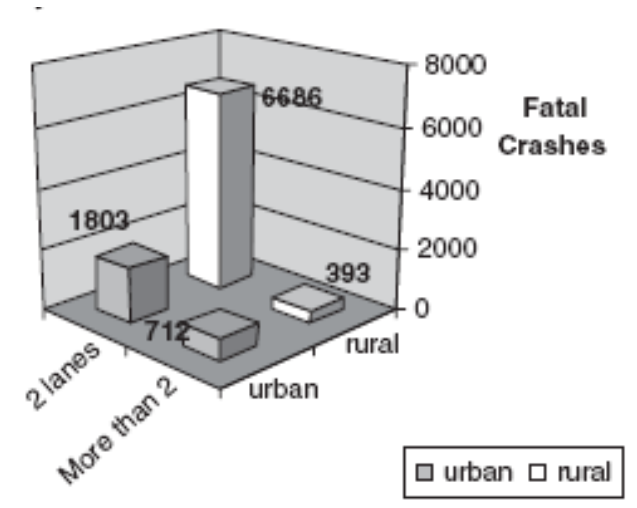

Fig.5: Fatal crashes on horizontal curves

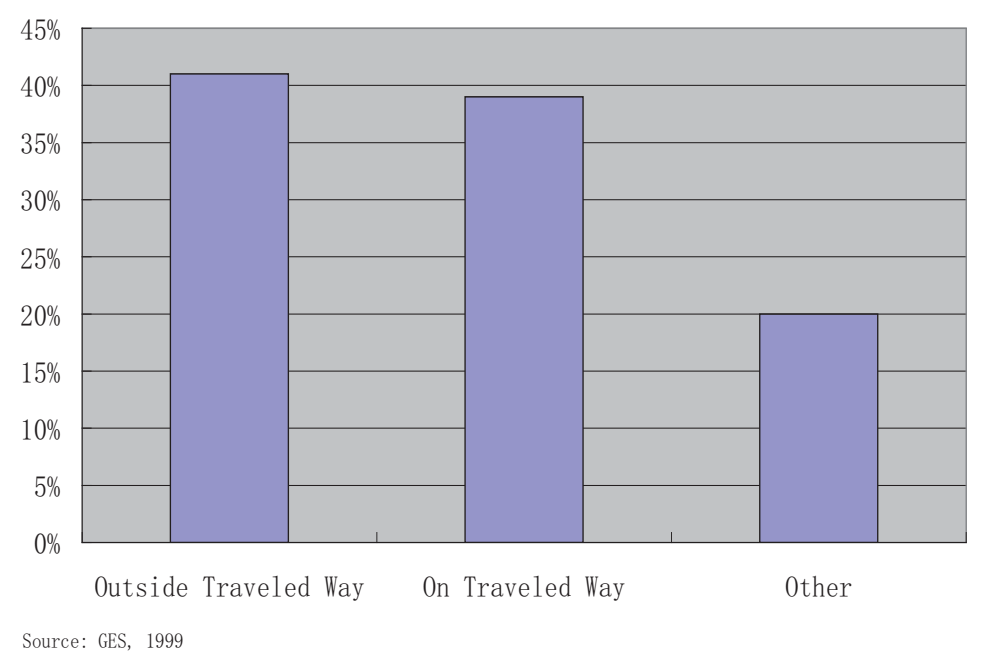

Fig.6: Position of crash relative to roadway all crashes on number of lanes and rural vs. urban by curved-roadway segments

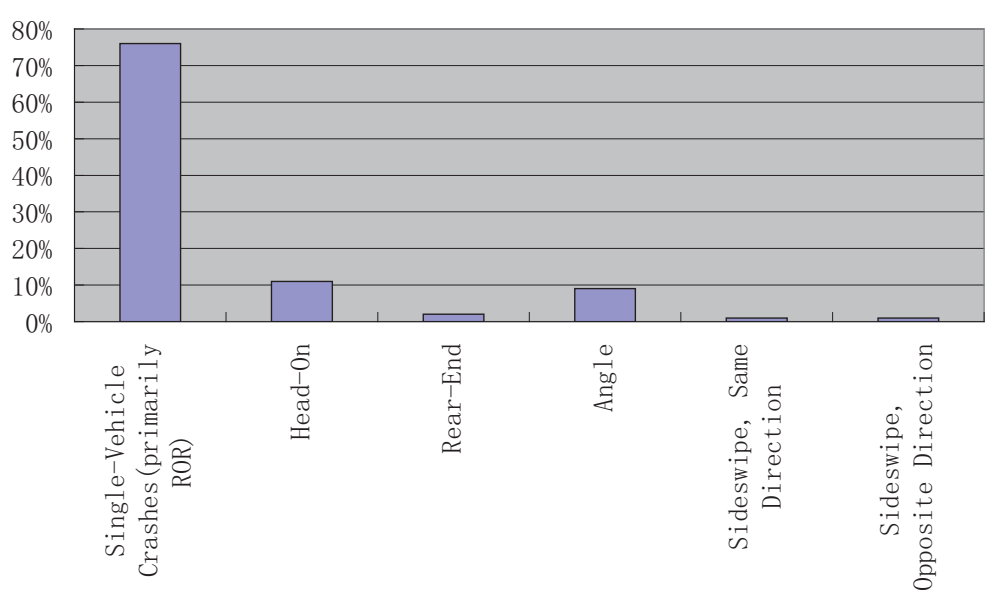

Fig.7: Percentage of curve-related fatal crashes by collision type

Thus, ROR and head-on crashes accounted for 87 percent of the fatal crashes at horizontal curves. Accordingly, the strategies for improving safety at horizontal curves focus on reducing the frequency and severity of these specific types of crashes.

Objectives of the Emphasis Area. The two main objectives for improving safety along horizontal curves are to

(1) $\square$ Reduce the likelihood of a vehicle leaving its lane and either crossing the roadway centerline or leaving the roadway at a horizontal curve and

(2) $\square$ Minimize the adverse consequences of leaving the roadway at a horizontal curve. 


\section{Objectives and Strategies for Improving Safety at Horizontal Curves}

Strategies designed to fulfill these objectives are presented. Because the strategic highway safety is geared toward low-cost, short-term safety improvements, strategies presented is arranged in general terms from low-cost, short-term treatments to high-cost, long-term treatments. Strategies classified by relative cost and time necessary for implementation

Objectives and Strategies 1. Objectives . Reduce the likelihood of a vehicle leaving its lane and either crossing the roadway centerline or leaving the roadway at a horizontal curve

3.1.2 Strategies

(1) Provide advance warning of unexpected changes in horizontal alignment

(2) Enhance delineation along the curve

(3) Provide adequate sight distance

(4) Install shoulder rumble strips

(5) Install centerline rumble strips

(6) Prevent edge dropoffs

(7) Provide skid-resistant pavement surfaces

(8) Provide grooved pavement

(9) Provide lighting of the curve

(10) Provide dynamic curve warning system

(11) Widen the roadway

(12) Improve or restore superelevation

(13) Modify horizontal alignment

(14) Install automated anti-icing systems

(15) Prohibit/restrict trucks with very long semitrailers on roads with horizontal curves that cannot accommodate truck off tracking

Objectives and Strategies 2. Objectives. Minimize the adverse consequences of leaving the roadway at a horizontal curve

Strategies.

(1) Design safer slopes and ditches to prevent rollovers

(2) Remove/relocate objects in hazardous locations

(3) Delineate roadside objects

(4) Add or improve roadside hardware

(5) Improve design and application of barrier and attenuation systems

\section{Conclusion}

The most prevalent types of crashes that occur on horizontal curves are ROR and head-on crashes; therefore, the emphasis is to reduce the frequency and severity of these types of crashes. These strategies may not eliminate crashes with other vehicles, pedestrians, bicyclists, and trains that may be directly in the path of the vehicle, but crash statistics do not indicate that these types of collisions are prevalent on curves. no distinction is made as to whether a strategy is more applicable at an isolated horizontal curve located between two long tangents or whether the strategy should be applied to horizontal curves located along curvilinear alignments. In general, all of the strategies have the potential to be effective in both instances. Similarly, all of the strategies may be used in combinations to improve safety. For example, if the horizontal alignment is modified to increase the radius of a curve, it may also be appropriate to enhance the delineation along the curve.

Management of safety on horizontal curves is a major challenge for highway agencies. Given these constraints, safety management of horizontal curves should be conducted making full use of available accident record systems.

In addition, agencies that cannot identify potential problems on horizontal curves by automated means should consider the use of other methods, including noting public complaints, skid marks, and damage to roadside hardware, trees, and utility poles. Where safety concerns related to horizontal curves are found, this paper provides a range of safety improvements that can be considered. 


\section{References}

[1] "Guidance for Implementation of the AASHTO Strategic Highway Safety Plan" Volume7-A Guide for Reducing Collisions on Horizontal Curves, 2004.

[2] DG Said,Y Hassan,AEHA Omar, Quantification and Utilization of Driver Path in Improving Design of Highway Horizontal Curves, Transportation Research Board Meeting, 2007.

[3] "National Cooperative Highway Research Program", November 2007, Transportation Research Board National Research Council. 